\title{
Analysis of Trajectory Entropy for Continuous Stochastic Processes at Equilibrium
}

\author{
Kevin R. Haas, ${ }^{\dagger}$ Haw Yang, ${ }^{*,+}$ and Jhih-Wei Chu* ${ }^{*}, \mathrm{ll}$, \\ ${ }^{\dagger}$ Department of Chemical and Biomolecular Engineering, University of California-Berkeley, Berkeley, California 94720, United \\ States \\ ${ }^{\ddagger}$ Department of Chemistry, Princeton University, Princeton, New Jersey 08544, United States \\ IIDepartment of Biological Science and Technology, National Chiao Tung University, Hsinchu 30068, Taiwan \\ ${ }^{\S}$ Institute of Bioinformatics and Systems Biology, National Chiao Tung University, Hsinchu 30068, Taiwan
}

ABSTRACT: The analytical expression for the trajectory entropy of the overdamped $d x_{t}=D F\left(x_{t}\right) d t+\sqrt{2 D} d W$ Langevin equation is derived via two approaches. The first route goes through the Fokker-Planck equation that governs the propagation of the conditional probability density, while the second method goes through the path integral of the OnsagerMachlup action. The agreement of these two approaches in the continuum limit underscores the equivalence between the partial differential equation and the path integral formulations for stochastic processes in the context of trajectory entropy. The

$$
\begin{aligned}
& \mathcal{S} \equiv-\int_{0}^{t_{\text {tos }}} \mathcal{D} X(t) \mathcal{P}[X(t)] \ln \frac{\mathcal{P}[X(t)]}{\mathcal{Q}[X(t)]} \\
& S_{\text {eq }}=-\int d x p_{\text {eq }}(x) \ln p_{\text {eq }}(x) \\
& \mathcal{S}[F(x), D]=S_{\text {eq }}-\frac{t_{\text {obs }}}{2}\left\langle D F^{2}(x)\right\rangle_{\text {eq }}+\lim _{t \rightarrow 0^{+}} \frac{t_{\text {obs }}}{\Delta t} \ln \sqrt{D}
\end{aligned}
$$
values obtained using the analytical expression are also compared with those calculated with numerical solutions for arbitrary time resolutions of the trajectory. Quantitative agreement is clearly observed consistently across different models as the time interval between snapshots in the trajectories decreases. Furthermore, analysis of different scenarios illustrates how the deterministic and stochastic forces in the Langevin equation contribute to the variation in dynamics measured by the trajectory entropy.

\section{INTRODUCTION}

Consider very generally a system that interacts with its environment and evolves over time. Let $x$ be some continuous order parameter of interest (or an experimental or computational observable) that characterizes the spatial configuration and represents the state of the system. For example, $x$ could be a molecule-scale observable in single-molecule experiments, such as the orientation-averaged dipole-dipole distance in a single-molecule Förster-type resonance energy transfer experiment, ${ }^{1}$ the constant-force extension in a laser tweezers/atomic force pulling experiment, ${ }^{2}$ or a collective coordinate from molecular dynamics simulations, ${ }^{3}$ even a state variable in a quantum-control experiment. ${ }^{4}$

In many problems of this type, including the examples given above, the time evolution of $x$ can be described by the overdamped Langevin equation, 5

$$
\mathrm{d} x_{t}=D F\left(x_{t}\right) \mathrm{d} t+\sqrt{2 D} \mathrm{~d} W_{t}
$$

where the subscript in $x_{t}$ is time. In the Langevin equation, $D$ is the diffusion coefficient specifying the magnitude of the stochastic forces modeled by the Wiener process, $\mathrm{d} W_{t}$, satisfying $\left\langle\mathrm{d} W_{t} \mathrm{~d} W_{t^{\prime}}\right\rangle=\delta\left(t-t^{\prime}\right) \mathrm{d} t$. On the other hand, the deterministic component of the Langevin equation comes from the potential of mean force (PMF), $V(x)$, and the mean force is $F(x)=-\mathrm{d} V(x) / \mathrm{d} x$. Here, we consider the PMF as nondimensionalized by $k_{\mathrm{B}} T$, where $k_{\mathrm{B}}$ is the Boltzmann constant and $T$ is the temperature.

Suppose in one realization that the system is at an initial configuration $x_{0}$ at time zero. Then propagating the Langevin equation will trace out a trajectory $X(t)$, a continuous but nondifferentiable function that gives a value of $x_{t}$ at time $t$, with $t$ varying between 0 and the finite period of observation, $t_{\mathrm{obs}}$. For stationary processes, the system reaches equilibrium and the probability density of obtaining a particular value of $X(t)=$ $x$ along the trajectory is given by $p_{\mathrm{eq}}(x)=\exp (-V(x)) / Z_{\mathrm{eq}}$, where $Z_{\mathrm{eq}}=\int \mathrm{d} x \exp (-V(x))$ is the equilibrium partition function. ${ }^{6}$ In this work, we consider an ensemble of trajectories realized at equilibrium with the probability density distribution of $x_{0}$ being $p_{\text {eq }}\left(x_{0}\right)$. This initial-state distribution in the trajectories is associated with the equilibrium entropy, $S_{\text {eq }}$, determined solely by the PMF as $S_{\mathrm{eq}}=-\int \mathrm{d} x p_{\mathrm{eq}}(x) \ln p_{\mathrm{eq}}(x)$ and contains no information regarding the dynamics. ${ }^{7-11}$ In addition to the variability in the trajectories due to $p_{\text {eq }}\left(x_{0}\right)$, stochastic forces in the Langevin equation would also make one observation of trajectory different from the others even when they have exactly the same starting configuration of time propagation. Therefore, a trajectory $X(t)$ is essentially a random variable.

A key question in the understanding of the system dynamics is thus how does the spread of trajectories depend on the parameters in the equation of motion. In this regard, we employ an entropy measure for the quantification:

Special Issue: James L. Skinner Festschrift

Received: January 31, 2014

Revised: $\quad$ April 27, 2014

Published: April 29, 2014 


$$
\mathcal{S} \equiv-\int_{0}^{t_{\mathrm{obs}}} \mathcal{D} X(t) \mathcal{P}[X(t)] \ln \frac{\mathcal{P}[X(t)]}{\mathcal{Q}[X(t)]}
$$

Here, $\mathcal{P}[X(t)]$ is the probability density of observing the trajectory $X(t), Q[X(t)]$ is the probability density of reaching the same observation via a reference dynamics, and the integration $\mathcal{D} X(t)$ is a path-integral over all continuous functions of $X(t)$ realizable from the system dynamics. The definition of trajectory entropy in eq 1 is in fact the negative of Kullback-Leibler (KL) divergence that represents the extra information required for encoding $\mathcal{P}[X(t)]$ relative to that for representing $Q[X(t)] . \mathcal{S}$ is thus negative and becomes zero only when the queried distribution is identical to that of the reference. $\mathrm{KL}$ divergence for entropy evaluation is often used to characterize the relaxation of nonequilibrium states back to equilibrium and the entropy production involved. ${ }^{12-14} \mathrm{KL}$ divergence has also been employed in studying dynamic trajectories for extracting information on reaction coordinates. $^{15,16}$

In a recent work, ${ }^{17}$ we illustrate that an analytical expression of $\mathcal{S}$ as a functional of the Langevin parameters can be obtained from the definition of eq 1 in the continuum limit of time resolution, $\Delta t \rightarrow 0^{+}$,

$$
\mathcal{S}[F(x), D]=S_{\mathrm{eq}}-\frac{t_{\mathrm{obs}}}{2}\left\langle D F^{2}(x)\right\rangle_{\mathrm{eq}}+\lim _{\Delta t \rightarrow 0^{+}} \frac{t_{\mathrm{obs}}}{\Delta t} \ln \sqrt{D}
$$

In that work, a path integral is used to directly evaluate the trajectory probability densities, for example, $\mathcal{P}[X(t)]$, as well as the trajectory entropy itself in eq 1 . Such an integration-based approach provides a transparent route to utilizing the statistical mechanics framework. The expectation operation, moment generation function, and other well-established tools are readily accessible through this machinery. A fully detailed derivation of eq 1 via this method is also included here to illustrate the stated merits. The path-integral approach of evaluating the trajectory entropy, however, does not lead to a straightforward way of conducting numerical analysis.

The primary objective of this paper is to provide an alternative derivation of eq 2 by relating the trajectory entropy to the entropy of the probability density of time propagation; that is, a differential equation-based approach. Fundamentally, this dual derivation underscores the equivalence of the pathintegral and differential-equation formulation of stochastic dynamics $^{18,19}$ via trajectory entropy. Following the differential-equation "based" analysis of the trajectory entropy also affords the implementation of very efficient numerical schemes for the evaluation of the trajectory entropy at arbitrary time resolutions. By combining the analytical and numerical analysis of trajectory entropy for several different dynamic models, we illustrate how the deterministic and stochastic components in the Langevin motion contribute to the observed variability and spread of equilibrium trajectories.

\section{DIFFERENTIAL-EQUATION APPROACH TO DERIVING THE TRAJECTORY ENTROPY}

The continuous function of a stochastic trajectory $X(t), t \in$ $\left[0, t_{\mathrm{obs}}\right]$, can be realized at specific instances in time separated by a time resolution $\Delta t$ to result in a segmented recording of $\mathbf{X}=$ $\left[x_{0}, x_{\Delta t}, x_{2 \Delta t}, \ldots, x_{t_{\mathrm{obs}}}\right]$. This vector has a spatial dimensionality of $N=t_{\text {obs }} / \Delta t$, in which the coordinates can be equivalently indexed as $\mathbf{X}=\left[x_{\tau} ; \tau=0, \ldots, N\right]$. The calculation of trajectory entropy via eq 1 can then be achieved by performing the evaluation first in this time segmentation form with an integration conducted at each time point of recording and then taking the limit of $\Delta t \rightarrow 0$. As such, the trajectory entropy is expressed as

$$
\mathcal{S}=\lim _{\Delta t \rightarrow 0}-\int \mathrm{d} x_{0} \int \mathrm{d} x_{1} \cdots \int \mathrm{d} x_{N} P(\mathbf{X}) \ln \frac{P(\mathbf{X})}{Q(\mathbf{X})}
$$

An important property of Markovian processes such as the Langevin dynamics is factorization of the probability density of trajectories with the conditional probability density of time propagation as $P(\mathbf{X})=p\left(x_{0}\right) \prod_{\tau=0}^{N} p\left(x_{\tau+1} \mid x_{\tau}\right)$. Applying the same factorization to the reference dynamics, $Q(X)=$ $q\left(x_{0}\right) \prod_{\tau=0}^{N} q\left(x_{\tau+1} \mid x_{\tau}\right)$, which is also assumed to be Markovian, we can simplify eq 3 to

$$
\begin{aligned}
\mathcal{S}= & -\int\left(\prod_{\tau^{\prime}=0}^{N} \mathrm{~d} x_{\tau^{\prime}} p\left(x_{0}\right) p\left(x_{\tau^{\prime}+1} \mid x_{\tau^{\prime}}\right)\right) \\
& \times\left(\ln \frac{p\left(x_{0}\right)}{q\left(x_{0}\right)}+\sum_{\tau=0}^{N} \ln \frac{p\left(x_{\tau+1} \mid x_{\tau}\right)}{q\left(x_{\tau+1} \mid x_{\tau}\right)}\right)
\end{aligned}
$$

Here, the $\lim _{\Delta t \rightarrow 0}$ action is dropped for now for clarity.

By grouping the logarithm terms in eq 4 and bringing the summation outside the integral, we can reorganize this equation to

$$
\begin{aligned}
\mathcal{S}= & -\sum_{\tau=0}^{N} \int \mathrm{d} x_{\tau+1} \mathrm{~d} x_{\tau} p\left(x_{\tau+1} \mid x_{\tau}\right) \\
& \ln \frac{p\left(x_{\tau+1} \mid x_{\tau}\right)}{q\left(x_{\tau+1} \mid x_{\tau}\right)}\left(\int \prod_{\substack{\tau^{\prime} \neq \tau \\
\tau^{\prime} \neq \tau+1}}^{N} \mathrm{~d} x_{\tau^{\prime}} p\left(x_{0}\right) p\left(x_{\tau^{\prime}+1} \mid x_{\tau^{\prime}}\right)\right) \\
& -\int \mathrm{d} x_{0}\left(\int \prod_{\tau^{\prime}}^{N} \mathrm{~d} x_{\tau^{\prime}} p\left(x_{0}\right) p\left(x_{\tau^{\prime}+1} \mid x_{\tau^{\prime}}\right)\right) \ln \frac{p\left(x_{0}\right)}{q\left(x_{0}\right)}
\end{aligned}
$$

For the products in this equation grouped by parentheses that do not contain the coordinates in the logarithms, integrations over the corresponding variables can be readily obtained via the relation of $\int \prod_{\tau^{\prime} \neq \tau, \tau+1}^{N} \mathrm{~d} x_{\tau^{\prime}} p\left(x_{0}\right) p\left(x_{\tau^{\prime}+1} \mid x_{\tau^{\prime}}\right)=p\left(x_{\tau}\right)$. In this work, we consider the stochastic process to be stationary. It can thus be recognized that the nontrivial integrals that remain are invariant to the choice of index $\tau$, leaving $N=t_{\mathrm{obs}} / \Delta t$ equivalent terms in the trajectory entropy:

$$
\begin{aligned}
\mathcal{S}= & -\frac{t_{\mathrm{obs}}}{\Delta t} \int \mathrm{d} x_{0} \mathrm{~d} x_{\Delta t} p\left(x_{\Delta t}, x_{0}\right) \ln \frac{p\left(x_{\Delta t} \mid x_{0}\right)}{q\left(x_{\Delta t} \mid x_{0}\right)} \\
& -\int \mathrm{d} x_{0} p\left(x_{0}\right) \ln \frac{p\left(x_{0}\right)}{q\left(x_{0}\right)}
\end{aligned}
$$

Although this result has been reached for different formatting of the dynamic process, ${ }^{20,21}$ the procedure is reproduced here for completion.

For the Langevin equation considered in this work, dynamics parameters in the equation of motion are time invariant, and because $p\left(x_{0}\right)=p_{\mathrm{eq}}(x)$, the marginal probability of finding the system at a specific state at a given time is the equilibrium probability density. Discarding the time-independent term coming from the entropy of the reference distribution (the -ln $q\left(x_{0}\right)$ part) because it only contributes an offset irrelevant to dynamics, we see that the second term in eq 6 is the equilibrium entropy, $S_{\text {eq }}$. The trajectory entropy defined in eq 1 
for systems that follow the Langevin equation is now simplified to

$$
\mathcal{S}=S_{\text {eq }}-\lim _{\Delta t \rightarrow 0} \frac{t_{\mathrm{obs}}}{\Delta t} \int \mathrm{d} x_{0} \mathrm{~d} x_{\Delta t} p\left(x_{\Delta t}, x_{0}\right) \ln \frac{p\left(x_{\Delta t} \mid x_{0}\right)}{q\left(x_{\Delta t} \mid x_{0}\right)}
$$

A similar expression can in fact be obtained for the trajectory probability density of Markovian processes in general, ${ }^{22}$ and the derivation for the continuous time and continuous space Langevin dynamics is shown here for completion.

The second term in eq 7 can be viewed as the entropy of the conditional probability density of time propagation and is defined as the Caliber by Jaynes. ${ }^{23-25}$ Caliber was proposed originally for finite-state Markov models as the conditional entropy of time propagation probabilities, $S(\Delta t)=-\sum_{i, j}^{N} \pi_{i} r_{i j} \log$ $r_{i, j}{ }^{26}$ The transition probability from state $i$ to state $j$ per unit of time $\Delta t$ is $r_{i, j}$ and the equilibrium probability of state $i$ is $\pi_{i}$. More details of this subject can be found in the recent review of Pressé and co-workers. ${ }^{21}$

Generalizing the caliber to the continuous space domain of the Langevin equation by letting $r_{i, j} \rightarrow p\left(x_{\Delta t} \mid x_{0}\right)$ and $\pi_{i} \rightarrow$ $p_{\text {eq }}\left(x_{0}\right)$ leads to the second term of eq 7 , except the ratio with respect to the reference probability density of time propagation, $q\left(x_{\Delta t} \mid x_{0}\right)$. Due to the nondifferentiability of the Weiner process in the Langevin equation, the conditional probability density of time propagation diverges at a rate of $\sim \ln (D \Delta t) \cdot{ }^{27,28}$ To overcome this difficulty, the $\mathrm{KL}$ divergence form of the caliber provides a way to secure a finite value for the trajectory entropy in the continuum limit of $\Delta t \rightarrow 0^{+}$. For example, we may choose the force-free Brownian dynamics with the same diffusion coefficient as the reference dynamics. Throughout this work, the reference dynamics model for calculating the trajectory entropy is set to the Brownian dynamics whose diffusion coefficient is labeled $D_{\text {ref }}$ and the second term in eq 7 is defined as $S_{\mathrm{KL}}(\Delta t)$ :

$$
S_{\mathrm{KL}}(\Delta t)=-\int \mathrm{d} x_{0} \mathrm{~d} x_{\Delta t} p\left(x_{\Delta t}, x_{0}\right) \ln \frac{p\left(x_{\Delta t} \mid x_{0}\right)}{q\left(x_{\Delta t} \mid x_{0}\right)}
$$

The trajectory entropy can thus be condensed into

$$
\mathcal{S}=S_{\text {eq }}+t_{\text {obs }} \lim _{\Delta t \rightarrow 0} \frac{S_{\mathrm{KL}}(\Delta t)}{\Delta t}
$$

Equation 9 is the starting point for deriving the analytical expression of the trajectory entropy. In the short time limit of $\Delta t \rightarrow 0^{+}$, the conditional probability densities of the Langevin and Brownian dynamics are equivalent in that one recovers the initial condition in both cases, that is, $p\left(x_{\Delta t} \mid x_{0}\right)=q\left(x_{\Delta t} \mid x_{0}\right)=$ $\delta\left(x_{\Delta t}-x_{0}\right)$. Consequently, the remaining expectation in eq 8 is taken over $\ln \left(\delta\left(x_{\Delta t}-x_{0}\right) / \delta\left(x_{\Delta t}-x_{0}\right)\right)=0$ as $\Delta t \rightarrow 0^{+}$and $\lim _{\Delta t \rightarrow 0} S_{\mathrm{KL}}(\Delta t)=0$. According to eq 9 , what remains to be developed is how $S_{\mathrm{KL}}(\Delta t)$ reacts when there is a competing factor of $\Delta t$ in the denominator in the continuum limit:

$$
\lim _{\Delta t \rightarrow 0} \frac{S_{\mathrm{KL}}(\Delta t)}{\Delta t}=\lim _{\Delta t \rightarrow 0}-\frac{\int \mathrm{d} x_{0} \mathrm{~d} x_{\Delta t} p\left(x_{\Delta t}, x_{0}\right) \ln \frac{p\left(x_{\Delta t} \mid x_{0}\right)}{q\left(x_{\Delta t} \mid x_{0}\right)}}{\Delta t}
$$

By application of the L'Hopitals rule, the central quantity for evaluating the trajectory entropy is identified to be

$$
\begin{aligned}
\lim _{\Delta t \rightarrow 0} \frac{S_{\mathrm{KL}}(\Delta t)}{\Delta t} & =-\left.\frac{\partial\left(\int \mathrm{d} x_{0} \mathrm{~d} x_{t} p\left(x_{t}, x_{0}\right) \ln \frac{p\left(x_{t} \mid x_{0}\right)}{q\left(x_{t} \mid x_{0}\right)}\right)}{\partial t}\right|_{t=0} \\
& =-\mathbb{E}_{p\left(x_{0}\right)}\left[\left.\frac{\partial}{\partial t}\left(\int \mathrm{d} x_{t} p\left(x_{t} \mid x_{0}\right) \ln \frac{p\left(x_{t} \mid x_{0}\right)}{q\left(x_{t} \mid x_{0}\right)}\right)\right|_{t=0}\right]
\end{aligned}
$$

Here, it is considered that $\Delta t=t-0$. In the last line of this equation, the $p\left(x_{0}\right)$ weighted integration over $x_{0}$ is represented by the shorthand notation of $\mathbb{E}_{p\left(x_{0}\right)}[\cdot]$. After the time derivatives are taken and the relation of $p\left(x_{t} \mid x_{0}\right)=q\left(x_{t} \mid x_{0}\right)=\delta\left(x_{t}-x_{0}\right)$ is applied in the zero time limit, this term becomes

$$
\lim _{\Delta t \rightarrow 0} \frac{S_{\mathrm{KL}}(\Delta t)}{\Delta t}=-\mathbb{E}_{p\left(x_{0}\right)}\left[\left.\int \mathrm{d} x_{t}\left(\frac{\partial p\left(x_{t} \mid x_{0}\right)}{\partial t}-\frac{\partial q\left(x_{t} \mid x_{0}\right)}{\partial t}\right)\right|_{t=0}\right]
$$

The time derivatives present in eq 12 are given by the Fokker-Plank equation (FPE) corresponding to Langevin dynamics: ${ }^{6}$

$$
\frac{\partial p\left(x_{t} \mid x_{0}\right)}{\partial t}=\frac{\partial}{\partial x_{t}}\left(D \frac{\partial p\left(x_{t} \mid x_{0}\right)}{\partial x_{t}}-D F\left(x_{t}\right) p\left(x_{t} \mid x_{0}\right)\right)
$$

To facilitate the calculation of eq 12 via eq 13 , a variable transformation using $\rho\left(x_{t}, x_{0}\right)=p\left(x_{t} \mid x_{0}\right)\left(p_{\text {eq }}\left(x_{0}\right) / p_{\text {eq }}\left(x_{t}\right)\right)^{1 / 2}$ is applied to both sides of eq 13. As a result, the FPE takes a symmetric form in terms of spatial derivatives:

$$
\frac{\partial \rho\left(x_{t}, x_{0}\right)}{\partial t}=D \frac{\partial^{2} \rho\left(x_{t}, x_{0}\right)}{\partial x_{t}{ }^{2}}-\left(\frac{F^{2}\left(x_{t}\right) D}{4}+\frac{F^{\prime}\left(x_{t}\right) D}{2}\right) \rho\left(x_{t}, x_{0}\right)
$$

The relation of $\left(p_{\mathrm{eq}}(x)\right)^{\prime}=F(x)\left(p_{\mathrm{eq}}(x)\right)$ is imposed in arriving at this equation. For the reference dynamics, the time derivative in eq 12 can be obtained from eq 14 by setting $F\left(x_{t}\right)$ to zero.

Therefore, for the case that the same diffusion coefficient is used for the reference dynamics and the process of interest, their second derivative terms of $\rho\left(x_{t}, x_{0}\right)$ cancel out each other. As a result, the calculation of trajectory entropy via eq 12 becomes

$$
\begin{aligned}
& \lim _{\Delta t \rightarrow 0} \frac{S_{\mathrm{KL}}(\Delta t)}{\Delta t} \\
& \quad=\mathbb{E}_{p_{\mathrm{eq}}\left(x_{0}\right)}\left[\int \mathrm{d} x_{t}\left(\frac{F^{\prime}\left(x_{t}\right) D}{2}+\frac{F^{2}\left(x_{t}\right) D}{4}\right) \delta\left(x_{t}-x_{0}\right)\right]
\end{aligned}
$$

Furthermore, the delta function initial condition in the zerotime continuum limit $\left(p\left(x_{t} \mid x_{0}\right) \rightarrow \delta\left(x_{t}-x_{0}\right)\right.$ as $\left.t \rightarrow 0\right)$ was applied in arriving at this equation. The integration over $x_{t}$ can thus be conducted trivially to give

$$
\begin{aligned}
\lim _{\Delta t \rightarrow 0} \frac{S_{\mathrm{KL}}(\Delta t)}{\Delta t} & =\mathbb{E}_{p_{\mathrm{eq}}\left(x_{0}\right)}\left[\frac{F^{\prime}\left(x_{0}\right) D}{2}+\frac{F^{2}\left(x_{0}\right) D}{4}\right] \\
& =\int \mathrm{d} x_{0} p_{\mathrm{eq}}\left(x_{0}\right)\left(\frac{F^{\prime}\left(x_{0}\right) D}{2}+\frac{F^{2}\left(x_{0}\right) D}{4}\right)
\end{aligned}
$$

Imposing integration by parts for the first term on the righthand side and recognizing that $\left(p_{\mathrm{eq}}(x)\right)^{\prime}=F(x)\left(p_{\mathrm{eq}}(x)\right)$ gives one of the main results of this work: 


$$
\lim _{\Delta t \rightarrow 0} \frac{S_{\mathrm{KL}}(\Delta t)[F(x), D ; D]}{\Delta t}=-\frac{D}{4}\left\langle F^{2}(x)\right\rangle_{\mathrm{eq}}
$$

In this equation, the condition of the reference $\mathrm{BD}$ for the functional of the Langevin parameters, $F(x)$ and $D$, is made explicit in $S_{\mathrm{KL}}(\Delta t)$ with $[F(x), D ; D]$. Since the deterministic force for $\mathrm{BD}$ is zero, it is not specified on the righthand side of the semicolon for simplicity. Finally, we can reach the conclusion via eq 9 that the trajectory entropy of Langevin dynamics referencing to $\mathrm{BD}$ has the following analytical expression:

$$
\mathcal{S}[F(x), D ; D]=S_{\mathrm{eq}}-t_{\mathrm{obs}} \frac{D}{4}\left\langle F^{2}(x)\right\rangle_{\mathrm{eq}}
$$

\section{INTEGRATION-BASED APPROACH TO DERIVING THE TRAJECTORY ENTROPY VIA THE ONSAGER-MACHLUP (OM) ACTION}

As shown in ref 17 , the result of eq 18 can also be obtained by performing path integral over the continuous functions with the integrand of the OM action of the Langevin equation. This approach does not require referring to the FPE. Therefore, the trajectory entropy provides an alternative platform for illustrating the equivalence between the partial differential equation and the path integral formulation of stochastic processes. For the completeness of comparing the two approaches of deriving the trajectory entropy, the procedure of evaluating the trajectory entropy via the OM action is briefly reconstructed here. The functional-derivative approach adopted in the following serves to deduce how the velocity-squared term in the $\mathrm{OM}$ action contributes to the trajectory entropy that is divergent in the continuum limit. The only assumption imposed here is that the initial states of trajectories follow the distribution at equilibrium.

The probability density of a trajectory $X(t)$ following the Langevin equation is described by the OM action: ${ }^{29-31}$

$$
\begin{aligned}
\mathcal{P}[X(t)]=\left(\mathrm{e}^{-V\left(x_{0}\right)} / Z_{\mathrm{eq}}\right)\left(\mathrm{e}^{-E^{\mathrm{OM}}[X(t)]} / \mathcal{Z}\right) \\
\mathcal{Z}=\int \mathcal{D} X(t) \mathrm{e}^{-E^{\mathrm{OM}}[X(t)]} \\
E^{\mathrm{OM}}[X(t)]=\frac{1}{2}\left(V\left(x_{t_{\mathrm{obs}}}\right)-V\left(x_{0}\right)\right) \\
\quad+\frac{1}{4} \int_{0}^{t_{\mathrm{obs}}} \mathrm{d} t\left(\frac{\dot{x}_{t}^{2}}{D}+D F^{2}\left(x_{t}\right)+2 D F^{\prime}\left(x_{t}\right)\right)
\end{aligned}
$$

Applying the above expression of $\mathcal{P}[X(t)]$ into the calculation of the trajectory entropy in eq 3 , imposing the $\mathrm{BD}$ reference, and discarding the scalar offsets due to $V_{\text {ref }}$ and $\left(Z_{\text {eq }}\right)_{\text {ref }}$ irrelevant to dynamics collecting terms leads to

$$
\begin{aligned}
& \mathcal{S}=\frac{1}{2}\left\langle V\left(x_{0}\right)+V\left(x_{t_{\mathrm{obs}}}\right)\right\rangle_{X(t)} \\
& +\frac{1}{4}\left\langle\int_{0}^{t_{\mathrm{obs}}} \mathrm{d} t D F^{2}\left(x_{t}\right)+2 D F^{\prime}\left(x_{t}\right)\right\rangle_{X(t)} \\
& +\frac{1}{4}\left\langle\int_{0}^{t_{\mathrm{obs}}} \mathrm{d} t \frac{\dot{x}_{t}^{2}}{D}-\frac{\dot{x}_{t}^{2}}{D_{\text {ref }}}\right\rangle_{X(t)}+\ln Z_{\text {eq }}+\ln \frac{\mathcal{Z}}{\mathcal{Z}_{\text {ref }}}
\end{aligned}
$$

In this equation, the dynamic parameters of reference dynamics are labeled with the subscript of "ref" to explicitly illustrate the term cancelation discussed later. Furthermore, we also employ the $\langle g[X(t)]\rangle_{X(t)}=\int \mathcal{D X}(t) \mathcal{P}[X(t)] g[X(t)]$ notation for an arbitrary functional of the trajectory $X(t)$, $g[X(t)]$. Because the path-integral expectation of single-time functions can be obtained by switching the order of integrating over time $\int \mathrm{d} t$ and path $\int \mathcal{D X X}(t),\left\langle\int \mathrm{d} t g\left(x_{t}\right)\right\rangle_{X(t)}$ becomes $\int \mathrm{d} t$ $\int \mathrm{d} x_{t} g\left(x_{t}\right) p_{\mathrm{eq}}\left(x_{t}\right)=t_{\mathrm{obs}}\langle g(x)\rangle_{\mathrm{eq}}{ }^{32}$. Therefore, the terms in eq 22 can reach the same factorization of the trajectory entropy as discussed in eq 6. Utilizing this fact with the definition of $\langle V(x)\rangle_{\mathrm{eq}}+\ln Z_{\mathrm{eq}}=S_{\mathrm{eq}}$ and integration by parts of $\left\langle F^{\prime}\left(x_{t}\right)\right\rangle_{\mathrm{eq}}=$ $-\left\langle F^{2}(x)\right\rangle_{\mathrm{eq}}$, eq 22 becomes

$$
\begin{aligned}
\mathcal{S}= & S_{\mathrm{eq}}-t_{\mathrm{obs}} \frac{D}{4}\left\langle F^{2}(x)\right\rangle_{\mathrm{eq}}+\frac{1}{4}\left\langle\int_{0}^{t_{\mathrm{obs}}} \mathrm{d} t \frac{\dot{x}_{t}^{2}}{D}-\frac{\dot{x}_{t}^{2}}{D_{\mathrm{ref}}}\right\rangle_{X(t)} \\
& +\ln \frac{\mathcal{Z}}{\mathcal{Z}_{\mathrm{ref}}}
\end{aligned}
$$

Although the velocity square term in eq 23 can be conveniently removed by having $D_{\text {ref }}=D$, it is instructive to retain this quantity here for revealing how the final result is achieved with an analytical expression of $\left\langle\dot{x}_{t}^{2}\right\rangle_{X(t)}$. To achieve this goal, the analytical expressions for $\mathcal{Z}$ and $\mathcal{Z}_{\text {ref }}$ are needed.

For $\mathrm{BD}$, the partition function of trajectories is ${ }^{6}$

$$
\mathcal{Z}_{\text {ref }}\left(D_{\text {ref }}\right)={\sqrt{4 \pi D_{\text {ref }} \Delta t}}^{\left(t_{\text {obs }} / \Delta t\right)}
$$

For the trajectory partition function of the Langevin dynamics, we probe $\mathcal{Z}$ by inspecting its functional derivative with respect to the mean force profile via eq 20 :

$$
\begin{aligned}
\frac{\delta \mathcal{Z}}{\delta F(y)} & =\frac{\delta}{\delta F(y)} \int \mathcal{D} X(t) \mathrm{e}^{-E_{\mathrm{OM}}[X(t)]} \\
& =-\int \mathcal{D} X(t) \frac{\delta E_{\mathrm{OM}}[X(t)]}{\delta F(y)} \mathrm{e}^{-E_{\mathrm{OM}}[X(t)]} \\
& =-\mathcal{Z} \int \mathcal{D} X(t) \frac{\delta E_{\mathrm{OM}}[X(t)]}{\delta F(y)} \mathcal{P}[X(t)] \\
& =-\mathcal{Z}\left\langle\frac{\delta E_{\mathrm{OM}}[X(t)]}{\delta F(y)}\right\rangle_{X(t)}
\end{aligned}
$$

Here, the functional derivative of the $\mathrm{OM}$ action can be obtained by the Euler-Lagrange equation:

$$
\begin{aligned}
\frac{\delta E_{\mathrm{OM}}[X(t)]}{\delta F(y)} & \\
= & \frac{D}{2} \int_{0}^{t_{\mathrm{obs}}} \mathrm{d} t\left(F\left(x_{t}\right) \delta\left(x_{t}-y\right)+\frac{\mathrm{d} \delta\left(x_{t}-y\right)}{\mathrm{d} x_{t}}\right) \\
& +\int \mathrm{d} x_{0}^{\prime} \delta\left(x_{0}^{\prime}-y\right)-\int \mathrm{d} x_{t_{\mathrm{obs}}^{\prime}} \delta\left(x_{t_{\mathrm{obs}}^{\prime}}^{\prime}-y\right)
\end{aligned}
$$

Therefore, the functional dependence of $E_{\mathrm{OM}}$ and $\mathcal{Z}$ on the mean force profile $F(x)$ is fully considered. Because the functional derivative contains only terms that are local in time, the $\int{ }^{x} \mathrm{~d} x^{\prime} \delta\left(x^{\prime}-y\right)$ terms are equivalent and cancel each other. Moreover, the resulting trajectory expectation reduces to an expectation over the equilibrium distribution: 


$$
\begin{aligned}
& \frac{\delta \mathcal{Z}}{\delta F(y)} \\
& \quad=-Z t_{\mathrm{obs}} \int \mathrm{d} x \frac{D}{2} p_{\mathrm{eq}}(x)\left(F(x) \delta(x-y)+\frac{\mathrm{d} \delta(x-y)}{\mathrm{d} x}\right)
\end{aligned}
$$

Applying integration by parts on the term containing the delta function derivative and recalling again that $\mathrm{d} p_{\mathrm{eq}}(x) / \mathrm{d} x=F(x)$ $p_{\text {eq }}(x)$ for the domain $x \in \mathbb{R}$ and recognizing that for the equilibrium probability density $p_{\mathrm{eq}} \mathrm{I}_{-\infty, \infty}=0$ to discard the boundary terms of $\left.F(x) p_{\mathrm{eq}}(x)\right|_{-L, L}=0$, we finally arrive at the cancellation of

$$
\frac{\delta \mathcal{Z}}{\delta F(y)}=-Z_{\mathrm{obs}} \frac{D}{2}(F(y)-F(y)) p_{\mathrm{eq}}(y)=0 .
$$

This means that the trajectory partition function is independent of the mean force. That is, $\mathcal{Z}$ is simply the trajectory partition function of the corresponding zero-force dynamics, that is, that of the reference Brownian dynamics in eq 24 :

$$
\mathcal{Z}(D)=\sqrt{4 \pi D \Delta t}^{\left(t_{\mathrm{obs}} / \Delta t\right)}
$$

This result is also consistent with the fact that it is the random forces in the Langevin equation, the same as those of $\mathrm{BD}$, that impose stochasticity in dynamics. The mean forces, on the other hand, give rise to deterministic behaviors.

Therefore, the term in eq 23 involving the ratio of the two trajectory partition functions is zero as long as the same diffusion coefficient is employed for both dynamics. Furthermore, the result of eq 29 also informs the velocity-squared terms in eq 23. Because $\mathcal{Z}$ is the cumulant generator of the $\mathrm{OM}$ action defined in eq 21 , taking $\mathrm{d}(\ln \mathcal{Z}) / \mathrm{d}(1 / D)$ to the righthand side of eq 29 and imposing eq 21 , one can obtain an expression for the path integral of the velocity square term as

$$
\left\langle\dot{x}_{t}{ }^{2}\right\rangle_{X(t)}=\frac{2 D}{\Delta t}-D^{2}\left\langle F^{2}(x)\right\rangle_{\mathrm{eq}}
$$

Indeed, the issue due to the nondifferentiability of Langevin trajectories on making the velocity-squared term diverging in the continuum limit can be removed by having $D_{\text {ref }}=D$. It is also clear that the result eq 17 and hence eq 18 can be derived through the route of analyzing the OM action by applying the results of eqs 29 and 30 to eq 23. The final expression is the result stated earlier in eq 2 .

\section{DEPENDENCE OF THE TRAJECTORY ENTROPY ON DIFFUSION COEFFICIENT}

By referencing to $\mathrm{BD}$ with the same diffusion coefficient, we show that the trajectory entropy takes the form of eq 18. This choice of setting the reference diffusion coefficient can in fact be relieved to allow $D_{\text {ref }}$ to assume an arbitrary value. The derivation and analytical expression presented in this section illustrate how the trajectory entropy of the Langevin equation depends on the diffusion coefficient.

After the explicit contribution of $F(x)$ to trajectory entropy in eq 18 is established, the remaining component of diffusion can be evaluated by comparing two stochastic processes with constant mean forces and diffusion coefficients although the values of one process can be different from another. $\mathrm{BD}$ is thus a special case that the constant force takes the zero value. For the two processes, letting the constant forces be $F$ and $F_{\text {ref }}$ and diffusion coefficients be $D$ and $D_{\text {ref }}$, we set the process designated by the subscript to be the reference dynamics for calculating the trajectory entropy. The probability density of time propagation of the constant-force diffusion in the zerotime limit is known as ${ }^{6}$

$$
p\left(x_{\Delta t} \mid x_{0}\right)=\frac{1}{\sqrt{4 \pi D \Delta t}} \exp \left(-\frac{\left(x_{\Delta t}-x_{0}-F D \Delta t\right)^{2}}{4 D \Delta t}\right)
$$

Here, both $\mathcal{P}[X(t)]$ and $\mathcal{Q}[X(t)]$ defined in eq 1 follow the functional form of eq 31 . The corresponding result of $\mathrm{BD}$ can be retrieved by setting $F=F_{\text {ref }}=0$ in the analytical expression derived via eq 31 . When eq 31 is applied to eq 8 , the twodimensional integral for evaluating the $S_{\mathrm{KL}}(\Delta t)$ is

$$
\begin{array}{r}
\int \mathrm{d} x_{0} p\left(x_{0}\right) \int \mathrm{d} x_{\Delta t} \frac{1}{\sqrt{4 \pi D t}} \exp \left(-\frac{\left(x_{\Delta t}-x_{0}-F D \Delta t\right)^{2}}{4 D t}\right) \\
\times\left[\frac{1}{2} \ln 4 D \Delta t-\frac{1}{2} \ln 4 D_{\mathrm{ref}} \Delta t+\frac{\left(x_{\Delta t}-x_{0}-F D \Delta t\right)^{2}}{4 D \Delta t}\right. \\
\left.-\frac{\left(x_{\Delta t}-x_{0}-F_{\mathrm{ref}} D_{\mathrm{ref}} \Delta t\right)^{2}}{4 D_{\mathrm{ref}} \Delta t}\right]
\end{array}
$$

After imposing the results of calculating the expected moments of Gaussian processes, $\left\langle x_{\Delta t}-x_{0}\right\rangle=F D \Delta t$ and $\left\langle\left(x_{\Delta t}-x_{0}\right)^{2}\right\rangle=$ $(F D \Delta t)^{2}+2 D \Delta t$, we arrive at

$$
\begin{aligned}
& \frac{S_{\mathrm{KL}}(\Delta t)\left[F, D ; F_{\mathrm{ref}}, D_{\mathrm{ref}}\right]}{\Delta t} \\
& =\frac{1}{\Delta t}\left[\frac{1}{2} \ln \left(\frac{D}{D_{\text {ref }}}\right)-\frac{1}{2}+\frac{D}{2 D_{\text {ref }}}\right]+\frac{\left(F D-F_{\text {ref }} D_{\text {ref }}\right)^{2}}{4 D_{\text {ref }}}
\end{aligned}
$$

Therefore,

$$
\frac{S_{\mathrm{KL}}(\Delta t)\left[D ; D_{\mathrm{ref}}\right]}{\Delta t}=\frac{1}{\Delta t}\left[\frac{1}{2} \ln \left(\frac{D}{D_{\mathrm{ref}}}\right)-\frac{1}{2}+\frac{D}{2 D_{\mathrm{ref}}}\right]
$$

Equation 34 is for two BD processes with different diffusion coefficients after dropping the force-containing term in eq 33 and can be employed for calculating trajectory entropy via eq 9 . In the continuum limit of $\Delta t \rightarrow 0^{+}$, the trajectory entropy thus diverges as $\propto 1 / \Delta t$ due to the stochastic diffusion in the Langevin equation.

In the coming section, the values of $S_{\mathrm{KL}}(\Delta t)\left[F(x), D ; D_{\text {ref }}\right]$ will be calculated numerically and compared with the results of combining the analytical expressions of eqs 17 and 34:

$$
\begin{aligned}
& \frac{S_{\mathrm{KL}}(\Delta t)\left[F(x), D ; D_{\mathrm{ref}}\right]}{\Delta t} \\
& =\frac{S_{\mathrm{KL}}(\Delta t)[F(x), D ; D]}{\Delta t}+\frac{S_{\mathrm{KL}}(\Delta t)\left[D ; D_{\mathrm{ref}}\right]}{\Delta t} \\
& \approx-\frac{D}{4}\left\langle F^{2}(x)\right\rangle_{\mathrm{eq}}+\frac{1}{\Delta t}\left[\frac{1}{2} \ln \left(\frac{D}{D_{\text {ref }}}\right)-\frac{1}{2}+\frac{D}{2 D_{\text {ref }}}\right]
\end{aligned}
$$

The third line of eq 35 is only strictly valid in the zero time limit. The manner by which it approaches the analytical expression will be demonstrated in the next section. 


\section{NUMERICAL ANALYSIS OF THE TRAJECTORY ENTROPY}

Without loss of generality, the three dynamics models with the mean force $F(x)$ and $P_{\mathrm{eq}}(x)$ profiles shown in Figure 1 are

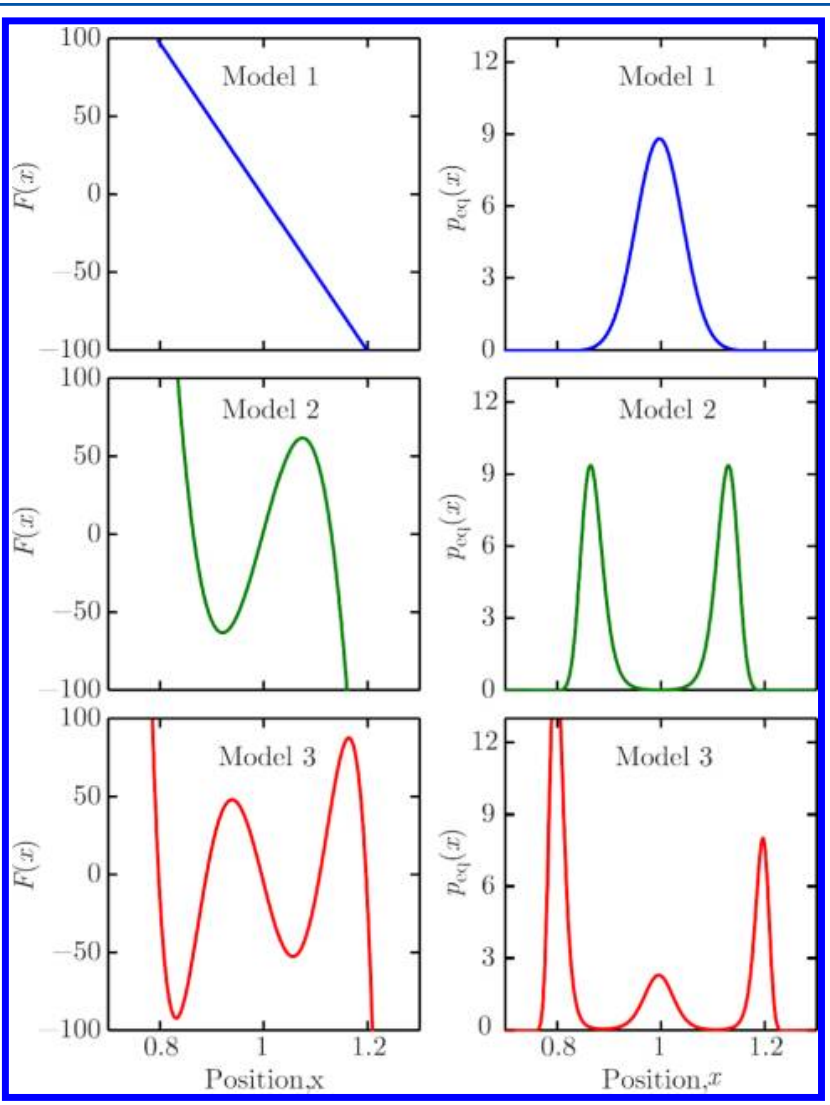

Figure 1. Profiles for the mean force and equilibrium probability density of the three model systems considered in this work. All physical variables are in dimensionless units.

considered for the comparison between analytical and numerical results. Model 1 has a single minimum in the PMF, while models 2 and have two and three minima, respectively. In the context of protein folding and protein conformational changes, these models may correspond to scenarios with different numbers of intermediate states. The PMFs shown in Figure 1 are also devised to have biologically relevant energetics of reaction barriers, $(5-10) k_{\mathrm{B}} T$, and identical values of equilibrium entropy. As such, the well of model 1 is wider than those of models 2 and 3 . This section will show that the resulting dynamics of the three models are different as characterized via the trajectory entropy (eq 9), despite their equivalence in equilibrium entropy.

To calculate the caliber via eq 8 , the FPE of the Langevin equation (eq 13) are solved to determine $p\left(x_{t} \mid x_{0}\right)$ with the initial condition $\left.p\left(x_{t} \mid x_{0}\right)\right|_{t=0}=\delta\left(x_{t}-x_{0}\right)$ and the zero-flux boundary conditions $J\left(x_{t}=L\right)=J\left(x_{t}=-L\right)=0$. The same initial and boundary conditions are also used to obtain $q\left(x_{t} \mid x_{0}\right)$ of the reference BD with an arbitrary diffusion coefficient. Our numerical solution of the FPE follows the procedure outlined in Gardiner, ${ }^{6}$ performing an eigen-decomposition of the time propagation operator of $p\left(x_{t} \mid x_{0}\right)$. Expressing the solution of eq 13 as $p(x, t)$ with the initial condition implied and dropping the subscript for the spatial variable, the eigen-decomposition uses a time dependent exponent with scale $\lambda$ and the spatial function $\Psi(x)$ :

$$
p(x, t)=\Psi(x) \mathrm{e}^{-\lambda t}
$$

The following eigen-value problem then emerges after applying this form of solution to the FPE:

$$
D \frac{\mathrm{d}^{2} \Psi(x)}{\mathrm{d} x^{2}}-\frac{\mathrm{d}(D F(x) \Psi(x))}{\mathrm{d} x}=\lambda \Psi(x)
$$

Although any numerical method for solving eigen-value problems can be applied, we obtained the family of eigenvectors $\psi_{i}(x)$ for eigenvalue $\lambda_{i}$ via a spectral finite element method, ${ }^{33}$ where the form of the eigenfunction is assumed to be a linear combination of low order polynomials $u_{j}(x)$ localized in spatially resolved elements.

$$
\psi_{i}(x)=\sum_{j} c_{i, j} u_{j}(x)
$$

Convergence of the numerical solution was reached with 500 elements of seventh order polynomials. The required number of elements and polynomial order in each element to reach convergence certainly depend on the PMF profile and $D$ value. Selection of the aforementioned level of numerical accuracy was conducted in a trial-and-error manner by ensuring convergence of all three models listed in Figure 1. This scheme allows numerical analysis of eq 35 to be very close to the continuum limit of $\Delta t \rightarrow 1 \times 10^{-7} \mathrm{~s}$.

Figure 2a shows the RMS (root-mean-square) difference of the first 128 eigenvalues $\left(\delta \lambda_{128}\right)$ with respect to the values obtained with 500 elements of seventh order polynomials. The behavior of spectral convergence ${ }^{33}$ of $\delta \lambda_{128}$ for the three models shown in Figure 1 is clearly seen in this figure.

The resulting set of eigenfunctions form a basis for the Fokker-Plank operator with the orthogonality property of

$$
\int \mathrm{d} x \Psi_{i}(x) \Psi_{j}(x) / p_{\text {eq }}(x)=\delta_{i, j}
$$

The general solution for the FPE is then a linear combination of eigenfunctions with coefficients $a_{i}$ :

$$
p(x, t)=\sum_{i} a_{i} \Psi_{i}(x) \mathrm{e}^{-\lambda_{i} t}
$$

The conditional probability density of time propagation, $p\left(x_{t}\right)$ $\left.x_{0}\right)$, is then found by resolving the initial condition of $p\left(x_{t} \mid\right.$ $\left.x_{0}\right)\left.\right|_{t=0}=\delta\left(x_{t}-x_{0}\right)$ :

$$
\sum_{i} a_{i} \Psi_{i}\left(x_{t}\right)=\delta\left(x_{t}-x_{0}\right)
$$

By application of the orthogonality property, $a_{i}$ is simply

$$
a_{i}=\int \mathrm{d} x_{t} \frac{\psi_{i}\left(x_{t}\right)}{p_{\mathrm{eq}}\left(x_{t}\right)} \delta\left(x_{t}-x_{0}\right)=\frac{\psi_{i}\left(x_{0}\right)}{p_{\mathrm{eq}}\left(x_{0}\right)}
$$

By applying eq 42 to eq 40 , we obtain the numerical solution for the FPE:

$$
p\left(x_{\Delta t} \mid x_{0}\right)=\sum_{i} \psi_{i}\left(x_{\Delta t}\right) \frac{\psi_{i}\left(x_{0}\right)}{p_{\mathrm{eq}}\left(x_{0}\right)} \exp \left(-\lambda_{i} \Delta t\right)
$$

The numerical solution is employed to calculate $S_{\mathrm{KL}}(\Delta t) / \Delta t$ via eq 8 and compared with the values provided by the analytical expression shown in eq 35. The convergence of $S_{\mathrm{KL}}$ at $\Delta t=$ 

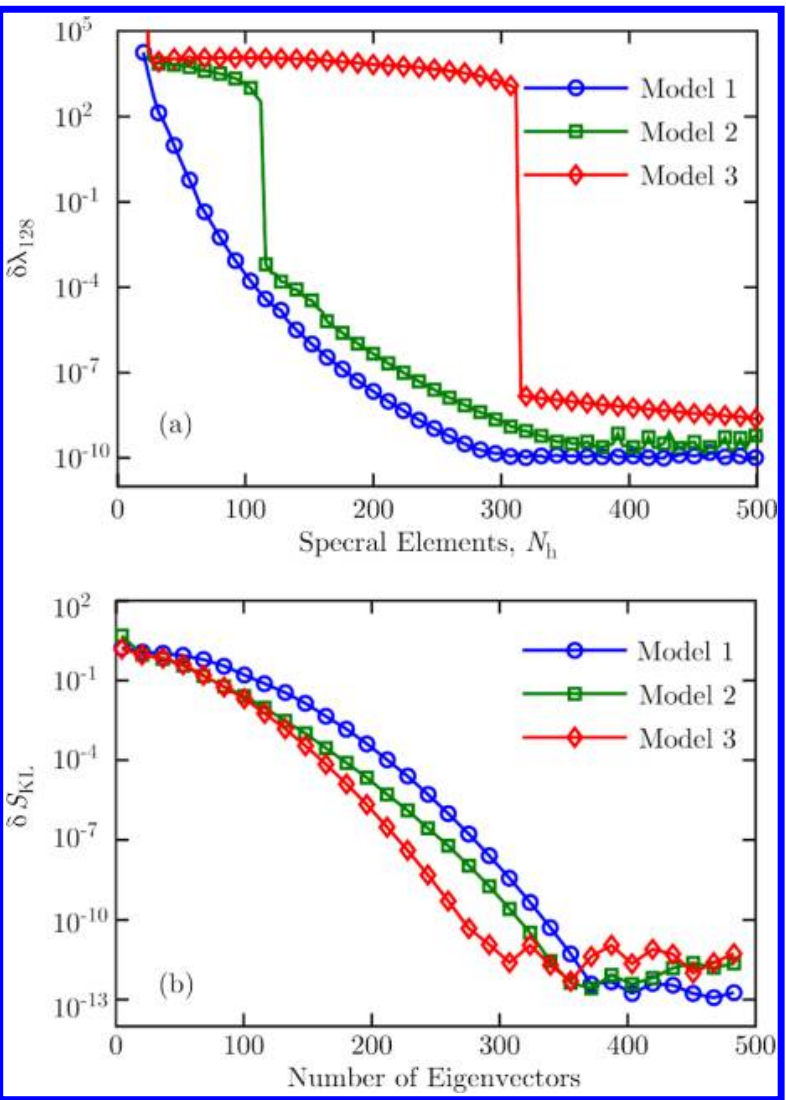

Figure 2. Convergence of the numerical calculations of $S_{\mathrm{KL}}(\Delta t)$. (a) The root-mean-square (RMS) difference of the first 128 eigenvalues, $\delta \lambda_{128}$, as a function of the spectral elements used in solving the FPE. The values of using 500 elements were used as the reference for comparison. All elements are represented via a seventh order polynomial. (b) The RMS difference of the calculated $S_{\mathrm{KL}}$ at $\Delta t=$ $10^{-5} \mathrm{~s}$ as a function of the number of eigenvectors used in the calculation. 500 elements of seventh polynomials were used for conducting the eigenbasis expansion.

$10^{-5} \mathrm{~s}$ with the number of eigenvalues used in the calculation is shown in Figure $2 \mathrm{~b}$. The values of $\delta S_{\mathrm{KL}}$ are the RMS differences with respect to the entropy calculation of using 500 elements of seventh order polynomials. In this convergence test, $D=D_{\text {ref }}=$ 1 in the dimensionless unit.

Figure 3 shows $S_{\mathrm{KL}}(\Delta t) / \Delta t$ as a function of $\Delta t$ for the three models in Figure 1 in the special case of $D=D_{\text {ref. }}$. The contribution from stochastic diffusion via the second term in the righthand side of eq 35 thus vanishes for the results based on the analytical expression. It can be seen from the numerical solutions that as $\Delta t$ increases, the differences in the three models due to dynamics disappear. The magnitudes of $S_{\mathrm{KL}}(\Delta t) / \Delta t$ also decrease with $\Delta t$, indicating the reduction of dynamics information with lowering of the time resolution of trajectory recording. The quantitative agreement between numerical and analytical solutions seen in the short time limit of Figure 3 validates eq 17.

When $D \neq D_{\text {ref }}$, the stochastic diffusion alone contributes to the value of $S_{\mathrm{KL}}(\Delta t) / \Delta t$ as eq 35 indicates. In Figure 4 , the values of $S_{\mathrm{KL}}(\Delta t) / \Delta t$ are shown for two such cases. As expected, the numerical values of $S_{\mathrm{KL}}(\Delta t) / \Delta t$ monotonically decrease with $\Delta t$. However, the profiles are highly nonlinear. The three models show clear differences at intermediate $\Delta t$ values and unite at the high and low time resolution limits. In

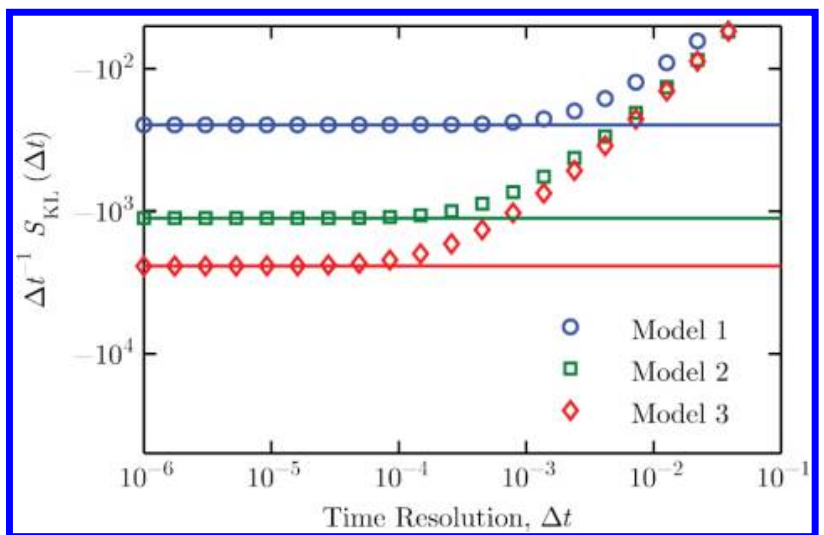

Figure 3. Values of $S_{\mathrm{KL}}(\Delta t) / \Delta t$ obtained by numerical calculations $(O, \square, \diamond)$ and the analytical expression of eq 35 (lines). For the three models shown in Figure 1, we employ $D=D_{\text {ref }}=2.0$ in this plot. All physical variables are nondimensionalized.

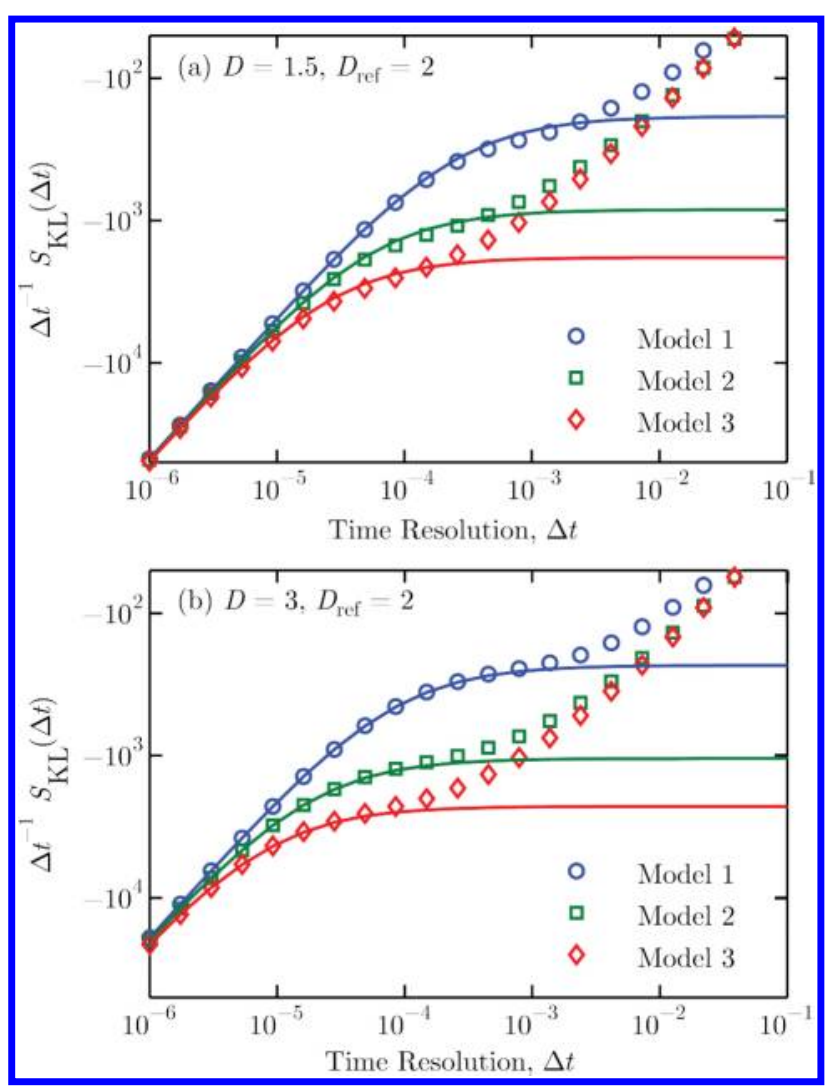

Figure 4. Values of $S_{\mathrm{KL}}(\Delta t) / \Delta t$ obtained by numerical calculations $(O, \square, \diamond)$ and the analytical expression of eq 35 (lines). For the three models shown in Figure 1, we employ $D=1.5, D_{\text {ref }}=2.0$ in panel a and $D=3, D_{\text {ref }}=2.0$ in panel b. All physical variables are nondimensionalized.

the limit of low time resolution, the conditional probability density of time propagation in eq 8 becomes $p_{\mathrm{eq}}(x)$ and all three models have the same $S_{\text {eq }}$ value as mentioned earlier. In the continuum limit of high time resolution, on the other hand, the divergence of the stochastic diffusion term dominates the value of $S_{\mathrm{KL}}(\Delta t) / \Delta t$ in eq 35 . Figure 4 shows that the numerical values of $S_{\mathrm{KL}}(\Delta t) / \Delta t$ of all three models converge as $\Delta t \rightarrow 0$ and provides a cross-validation of eq 34 .

Figure 4 also shows that the analytical expression of eq 35 can describe $S_{\mathrm{KL}}(\Delta t) / \Delta t$ over a significant range in the finite $\Delta t$ 
regime. Model 3 is the most complex of the three models considered here; its profile shows the earliest onset of deviation between the analytical and numerical values among the three. Appearance of this discrepancy for model 2 occurs at a larger $\Delta t$, and that for model 1 is the latest because it has the least complexity of the three, having only one well in the PMF. For the case with different values of the reference diffusion, $D_{\text {ref }}$, the time scale at which eq 35 starts to deviate from the numerical solution varies. On the other hand, the $D=D_{\text {ref }}$ option eliminates the contribution of eq 34 to $S_{\mathrm{KL}}(\Delta t) / \Delta t$, and the quantitative distinction of the three models becomes clear in Figure 3 in the continuum limit. This analysis illustrates that the $\mathrm{KL}$ divergence relative to a reference dynamics can be used to overcome the issue of stochastic divergence in the continuum limit for evaluating the trajectory entropy.

Another approach of removing the arbitrariness in the values of $S_{\mathrm{KL}}(\Delta t) / \Delta t$ due to the BD reference is letting $D_{\text {ref }}$ to go to infinity. With $D / D_{\text {ref }}$ approaching zero and the constant becoming negligible in this limit, only the $\ln D$ term in eq 34 contributes to the trajectory entropy. The universal inverse scaling of $S_{\mathrm{KL}}(\Delta t) / \Delta t$ with respect to $\Delta t$ in using large values of $D_{\text {ref }}$ can be seen in Figure 5 , in which the values of $S_{\mathrm{KL}}(\Delta t) / \Delta t$

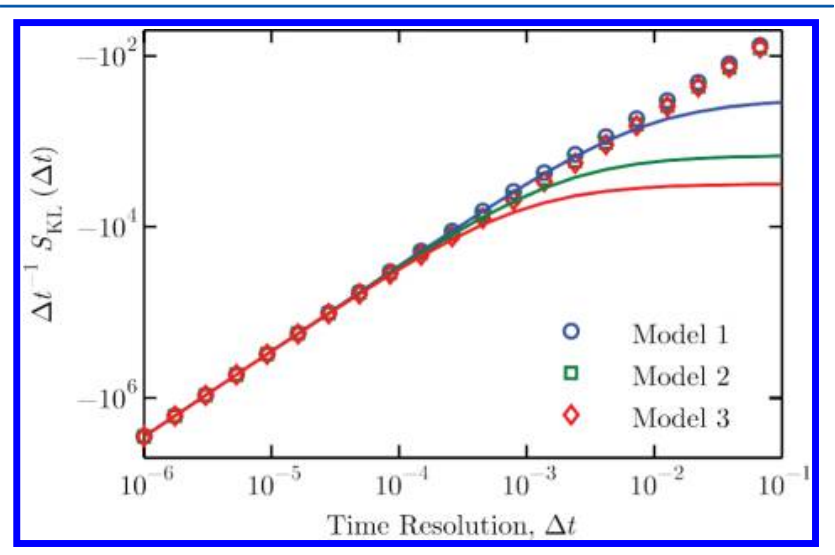

Figure 5. Values of $S_{\mathrm{KL}}(\Delta t) / \Delta t$ obtained by numerical calculations $(O, \square, \diamond)$ and the analytical expression of eq 35 (lines). For the three models shown in Figure 1, we employ $D=1.3$ and $D_{\text {ref }}=1000.0$ in this plot. All physical variables are nondimensionalized.

for the three model systems at $D=1.3$ and $D_{\text {ref }}=1000$ are presented to illustrate the behaviors of using a large $D_{\text {ref }}$. Therefore, after discarding the scaling constant of $D_{\text {ref }}$ in this approach of choosing the reference dynamics, the contribution of stochastic diffusion to the trajectory entropy in the continuous time limit has the functional form of $\ln \sqrt{ } D / \Delta t$.

\section{CONCLUSION}

In this work, we show that the trajectory entropy expression for the Langevin equation can be derived through two alternative routes via analyzing the partial differential equation of the time propagation as well as the path integral over all continuous functions of realizable trajectories. The equivalence of the path integral and partial differential equation formulation of stochastic dynamics is thus observed in the evaluation of the trajectory entropy. The derivation is conducted in the continuum limit at which the interval of trajectory recording is approaching zero. Although the conditional probability density of time propagation converges to the delta function initial condition, $p\left(x_{\Delta t} \mid x_{0}\right)=\delta\left(x_{\Delta t}-x_{0}\right)$ as $\Delta t \rightarrow 0^{+}$, we show that the rate of change of $p\left(x_{\Delta t} \mid x_{0}\right)$ dictates how the information on dynamics is encoded in trajectories. The derivation is based on the fact that the entropy of the probability density of time propagation, or the caliber, is the rate-of-change element of the trajectory entropy as shown in eq 9. In addition to the contribution of the deterministic mean forces in the Langevin equation represented via eq 17, an expression of eq 34 is also developed for quantifying how random diffusion alone adds to the values of the trajectory entropy. Therefore, our framework of analysis can be used to systematically extract how the deterministic and stochastic forces of the equation of motion affect dynamics. The combined expression of eq 35 also allows a direct comparison of the values of entropy obtained via numerical calculations and those according to the analytical formula. In the continuum limit, we show that the numerical results approach the analytical expression. Through the numerical studies, we also show how the different features in the potential of mean force may manifest themselves in the dynamics, giving rise to different extents of complexity. This quantitative agreement also serves as a way of validating the analytical results developed in this work. Even though the three model systems have identical levels of complexity in their static distributions in the sense that the equilibrium entropy calculation from $p_{\mathrm{eq}}(x)$ has identical values for these models, trajectory entropy can be used to reveal the differences in dynamics. For biomolecular systems and others for which the Langevin equation is employed for modeling the dynamics, the results of this work provide a theoretical foundation for quantitative analysis of data, including adopting the methods of statistical learning, ${ }^{34}$ and for development of dynamic models. ${ }^{35}$

\section{AUTHOR INFORMATION}

\section{Corresponding Authors}

*E-mail: hawyang@princeton.edu.

*E-mail: jwchu@nctu.edu.tw.

\section{Notes}

The authors declare no competing financial interest.

\section{ACKNOWLEDGMENTS}

The authors thank the reviewers for their helpful commentary and assistance in improving this manuscript. This work was supported by the University of California, Berkeley, Princeton University, and the National Chiao Tung University, Taiwan. The funding from the Ministry of Science and Technology of Taiwan, Republic of China, via Grant Number 102-2113-M009-022-MY2 is acknowledged.

\section{REFERENCES}

(1) Hanson, J. A.; Duderstadt, K.; Watkins, L. P.; Bhattacharyya, S.; Brokaw, J.; Chu, J.-W.; Yang, H. Illuminating the Mechanistic Roles of Enzyme Conformational Dynamics. Proc. Natl. Acad. Sci. U.S.A. 2007, 104, 18055-18060.

(2) Hummer, G.; Szabo, A. Free Energy Profiles from Singlemolecule Pulling Experiments. Proc. Natl. Acad. Sci. U.S.A. 2010, 107, 21441-21446.

(3) Schlick, T.; Collepardo-Guevara, R.; Halvorsen, L. A.; Jung, S.; Xiao, X. Biomolecular Modeling and Simulation: a Field Coming of Age. Q. Rev. Biophys. 2011, 44, 191-228.

(4) Kastoryano, M. J.; Wolf, M. M.; Eisert, J. Precisely Timing Dissipative Quantum Information Processing. Phys. Rev. Lett. 2013, 110, No. 110501.

(5) Seifert, U. Stochastic Thermodynamics, Fluctuation Theorems, and Molecular Machines. Rep. Prog. Phys. 2012, 75, No. 126001. 
(6) Gardiner, C. W. Handbook of Stochastic Methods for Physics, Chemistry, and the Natural Sciences; Springer Verlag: Berlin, 2004.

(7) Tomé, T.; de Oliveira, M. J. Entropy Production in Irreversible Systems Described by a Fokker-Planck Equation. Phys. Rev. E 2010, 82, No. 021120.

(8) Latora, V.; Baranger, M. Kolmogorov-Sinai Entropy Rate versus Physical Entropy. Phys. Rev. Lett. 1999, 82, 520-523.

(9) Pattanayak, A. Lyapunov Exponents, Entropy Production, and Decoherence. Phys. Rev. Lett. 1999, 83, 4526-4529.

(10) Kleeman, R. Information Theory and Dynamical System Predictability. Entropy 2011, 13, 612-649.

(11) Plastino, A. R.; Plastino, A. Non-extensive statistical mechanics and generalized Fokker-Planck equation. Phys. A (Amsterdam, Neth.) 2002, 222, 347-354.

(12) Bach, A.; Dürr, D. Entropy Density in Function Space and the Onsager-Machlup Function. Phys. Lett. A 1978, 69, 244-246.

(13) Roldán, E.; Parrondo, J. Estimating Dissipation from Single Stationary Trajectories. Phys. Rev. Lett. 2010, 105, No. 150607.

(14) Roldán, É.; Parrondo, J. M. R. Entropy Production and Kullback-Leibler Divergence between Stationary Trajectories of Discrete Systems. Phys. Rev. E 2012, 85, No. 031129.

(15) Sivak, D. A.; Crooks, G. E. Thermodynamic Metrics and Optimal Paths. Phys. Rev. Lett. 2012, 108, No. 190602.

(16) Peters, B.; Bolhuis, P. G.; Mullen, R. G.; Shea, J.-E. Reaction Coordinates, One-dimensional Smoluchowski Equations, and a Test for Dynamical Self-consistency. J. Chem. Phys. 2013, 138, No. 054106.

(17) Haas, K. R.; Yang, H.; Chu, J.-W. Trajectory Entropy of Continuous Stochastic Processes at Equilibrium. J. Phys. Chem. Lett. 2014, 5, 999-1003.

(18) Schuss, Z. Theory and Applications of Stochastic Differential Equations; Wiley: New York, 1980.

(19) Hummer, G.; Szabo, A. Free energy Reconstruction from Nonequilibrium Single-molecule Pulling Experiments. Proc. Natl. Acad. Sci. U.S.A. 2001, 98, 3658.

(20) Haken, H. A New Access to Path Integrals and Fokker Planck Equations via the Maximum Calibre Principle. Z. Phys. B: Condens. Matter 1986, 63, 505-510.

(21) Pressé, S.; Ghosh, K.; Lee, J.; Dill, K. A. Principles of Maximum Entropy and Maximum Caliber in Statistical Physics. Rev. Mod. Phys. 2011, 85, 1115-1141.

(22) Pantazis, Y.; Katsoulakis, M. A. A Relative Entropy Rate Method for Path Space Sensitivity Analysis of Stationary Complex Stochastic Dynamics. J. Chem. Phys. 2013, 138, No. 054115.

(23) Gaspard, P. Time-reversed Dynamical Entropy and Irreversibility in Markovian Random Processes. J. Stat. Phys. 2004, 117, 599615.

(24) Gómez-Gardeñes, J.; Latora, V. Entropy Rate of Diffusion Processes on Complex Networks. Phys. Rev. E 2008, 78, No. 065102.

(25) Ekroot, L.; Cover, T. M. The Entropy of Markov Trajectories. IEEE Trans. Inf. Theory 1993, 39, 1418-1421.

(26) Jaynes, E. T. The Minimum Entropy Production Principle. Annu. Rev. Phys. Chem. 1980, 31, 579-601.

(27) Paluš, M. On Entropy Rates of Dynamical Systems and Gaussian Processes. Phys. Lett. A 1997, 227, 301-308.

(28) Luschgy, H.; Pagès, G. Sharp Asymptotics of the Kolmogorov Entropy for Gaussian Measures. J. Funct. Anal. 2004, 212, 89-120.

(29) Onsager, L.; Machlup, S. Fluctuations and irreversible processes. Phys. Rev. 1953, 91, 1505-1512.

(30) Adib, A. B. Stochastic Actions for Diffusive Dynamics: Reweighting, Sampling, and Minimization. J. Phys. Chem. B 2008, 112, 5910-5916.

(31) Langouche, F.; Roekaerts, D.; Tirapegui, E. Functional Integration and Semiclassical Expansions; D. Reidel Pub. Co.: Dordrecht, the Netherlands, 1982.

(32) Turgeman, L.; Carmi, S.; Barkai, E. Fractional Feynman-Kac Equation for Non-Brownian Functionals. Phys. Rev. Lett. 2009, 103, No. 190201.

(33) Pozrikidis, C. Introduction to Finite And Spectral Element Methods Using Matlab; CRC Press: Boca Raton, FL, 2005.
(34) Haas, K. R.; Yang, H.; Chu, J.-W. Expectation-Maximization of the Potential of Mean Force and Diffusion Coefficient in Langevin Dynamics from Single Molecule FRET Data Photon by Photon. J. Phys. Chem. B 2013, 117, 15591-15605.

(35) Haas, K. R.; Yang, H.; Chu, J.-W. Fisher Information Metric for the Langevin Equation and Least Informative Models of Continuous Stochastic Dynamics. J. Chem. Phys. 2013, 139, No. 121931. 